\title{
A phase II study of docetaxel weekly in combination with carboplatin every three weeks as first line chemotherapy in stage IIB-IV epithelial ovarian cancer: Neurological toxicity and quality-of-life evaluation
}

\author{
BENGT SORBE ${ }^{1}$, MARIANNE GRAFLUND ${ }^{1}$, LISA NYGREN $^{1}$, GYÖRGY HORVATH $^{2}$, MARIE SWAHN $^{2}$, \\ KARIN BOMAN ${ }^{3}$, RENÉ BANGSHÖJ ${ }^{4}$, MARGARETA LOOD ${ }^{4}$ and HENRIC MALMSTRÖM ${ }^{5}$ \\ ${ }^{1}$ Department of Gynecological Oncology, University Hospital, Örebro; ${ }^{2}$ Department of Gynecological Oncology, \\ Sahlgrenska University Hospital, Gothenburg; ${ }^{3}$ Department of Gynecological Oncology, University \\ Hospital, Umeå; ${ }^{4}$ Department of Gynecology and Obstetrics, Central Hospital, Karlstad; \\ ${ }^{5}$ Department of Oncology, University Hospital, Linköping, Sweden
}

Received September 7, 2011; Accepted October 21, 2011

DOI: $10.3892 /$ ijo.2011.1286

\begin{abstract}
The purpose of this study was to assess the response rate, toxicity, progression-free survival (PFS) and overall survival (OS) in a series of advanced stage ovarian carcinoma patients treated with a first-line weekly docetaxel and three weekly carboplatin regimens. All eligible patients were treated with intravenous docetaxel $\left(30 \mathrm{mg} / \mathrm{m}^{2}\right)$ on Days 1,8 and 15 , and carboplatin (area under the curve, 5) on Day 1; Q21 days for at least 6 cycles. Neurological tests, questionnaires, and the EORTC QLQ-C30 and OV28 were used for quality-of-life assessments. One hundred and six patients received at least one cycle of primary chemotherapy (median 6.0; range, 1-9) and they were evaluable for toxicity assessment. Eighty-five patients had evaluable disease and received at least 3 courses of chemotherapy and were evaluable for clinical response rate. The overall response rate was $78.8 \%$ (95\% CI 70.1-87.5\%) and the biochemical response was $92.8 \%$ (95\% CI 87.2-98.4\%). The median PFS was 12.0 months and the median OS was 35.3 months. Thirty-six patients $(34.0 \%)$ experienced grades 3 and 4 neutropenia, which resulted in the removal of 3 patients. Six patients $(5.7 \%)$ experienced grades 3 or 4 thrombocytopenia. No patients experienced grade 3-4 sensory neuropathy. Epiphora, nail changes and fatigue were frequently recorded non-hematological side effects. The tolerable hematological toxicity (no need for colony-stimulating factors) and the low rate of severe neurotoxicity (only grade 1-2) and response
\end{abstract}

Correspondence to: Professor Bengt Sorbe, Department of Gynecological Oncology, University Hospital, S-701 85 Örebro, Sweden E-mail: bengt.sorbe@orebroll.se

Key words: ovarian cancer, docetaxel, carboplatin, weekly administration, neurological side effects, quality of life rates in line with the standard 3-week paclitaxel-carboplatin regimen for advanced primary ovarian carcinoma after suboptimal cytoreductive surgery make this regimen an interesting alternative in selected patients.

\section{Introduction}

Ovarian cancer is the seventh most common cancer in women worldwide, accounting for nearly 225,000 new cases and 140,000 deaths annually (1). Despite the efficacy of first-line chemotherapy comprising paclitaxel and carboplatin, nearly $70 \%$ of patients will eventually die due to complications related to the disease.

Investigations of new chemotherapy agents and alternative dosing schedules have been undertaken to prolong duration of responses and survival rates and to increase tolerability (2-5). Standard 3-week dosing schedules have been associated with improved response rates and progression-free survival, but still with disappointing long-term overall survival rates and relapses exceeding $70 \%$ (3).

Studies have analyzed the efficacy of primary therapy, but so far, data have not yet shown superiority of a specific standard triplet chemotherapy regimen for the treatment of ovarian carcinoma (3).

Weekly chemotherapy regimens have also been evaluated with regard to improved prognosis and reduced or altered drug toxicity. Promising activity and a favorable toxicity profile has been reported $(4,5)$. Higher total dose of paclitaxel (dose-dense) can be achieved with weekly regimens and theoretically, may be superior to the standard 3-week schedule for first-line therapy (6).

Docetaxel is an alternative to paclitaxel in combination with a platinum agent in first-line chemotherapy but also for treatment of recurrent ovarian cancer $(7,8)$. Standard 3 -week schedule seems to be of comparable efficacy with paclitaxel but with a different toxicity profile (9). More dose-dense weekly schedules have also been studied in small patient 
series of recurrent ovarian cancer (10-16). Results have been promising but further studies are warranted.

The purpose of the present study was for the first time to evaluate the response rate, progression-free survival and the toxicity profile of a novel chemotherapy regimen comprising weekly docetaxel and carboplatin administered every three weeks for first-line treatment of advanced-stage ovarian carcinoma. Special attention was paid at neurological toxicity and quality-of-life in this study.

\section{Patients and methods}

Eligibility. This was a phase II, prospective, multicenter study, including patients with histologically confirmed epithelial ovarian carcinoma from 6 gynecological oncology departments in Sweden. All patients were in FIGO stage IIB-IV and underwent primary cytoreductive surgery. The period of recruitment was from June 2004 to December 2008. In all, 110 patients were included in the study and 106 patients fulfilled inclusion and exclusion criteria and were evaluable for tumor response and or toxicity. Four patients were wrongly included with other diagnoses than ovarian carcinoma. Ninety patients (85\%) completed 3 or more courses of chemotherapy. Clinical and biochemical response rates were based on 85 patients with measurable disease and or elevated CA-125 levels at start of chemotherapy, but toxicity was recorded in all 106 patients $(\geq 1$ course of chemotherapy). After primary surgery 20 patients (19\%) were macroscopically free of tumor, 32 patients $(30 \%)$ were left with a residual carcinoma of $<2 \mathrm{~cm}$ and 54 patients (51\%) with a residual carcinoma $\geq 2 \mathrm{~cm}$. Patient and tumor characteristics are shown in Table I.

All patients received a chemotherapy regimen of weekly docetaxel $30 \mathrm{mg} / \mathrm{m}^{2}$ (0.5-h infusion) and carboplatin AUC 5 given every 3 weeks (17). Six cycles were administered during 18 weeks. At the discretion of the physician another 3 cycles of carboplatin were allowed. Sixty-eight patients (64\%) completed at least 6 cycles of chemotherapy. The median number of cycles was 6.0 (range 1-9). The mean dose intensity of docetaxel was $26.6 \mathrm{mg} / \mathrm{m}^{2} /$ week (95\% CI 25.7-27.6) and of carboplatin $103.1 \mathrm{mg} / \mathrm{m}^{2} /$ week (95\% CI 98.5-107.7).

Eligible patients had adequate bone marrow, renal and hepatic function, and had absolute neutrophil count $\geq 1.5 \times 10^{9} / 1$, platelet count $\geq 100 \times 10^{9} / 1$, serum-creatinine of $<1.25$ times normal, serum AST/ALT level of $<1.5$ times normal, no previous history of chemotherapy or radiotherapy and an Eastern Cooperative Oncology Group (ECOG) performance status $<2$. Exclusion criteria included severe infection, hypertension and myocardial infarction within previous 6 months, congestive heart failure, prior serious allergic reactions and previous malignancy within 5 years. The study was approved by the Ethics Committee of the University of Linköping and by all participating centers (Dnr 03-258).

Drug administration. Patients were treated with intravenous docetaxel $\left(30 \mathrm{mg} / \mathrm{m}^{2}\right)$ and carboplatin [area under the curve (AUC), 5] on Day 1. Docetaxel was repeated on Days 8 and 15 and was administered via half-hour infusion and the carboplatin was given in accordance with the Calvert formula (17) for $30 \mathrm{~min}$ on Day 1. Before docetaxel infusion, patients were premedicated with intravenous dexamethasone, diphen-
Table I. Patient characteristics for the trial weekly docetaxelcarboplatin $(\mathrm{n}=106)$.

\begin{tabular}{|c|c|}
\hline Characteristics & Data \\
\hline Mean age, years (range) & $63.3(28-80)$ \\
\hline \multicolumn{2}{|l|}{ Histological type, n (\%) } \\
\hline Papillary serous & $86(81.1)$ \\
\hline Mucinous & $2(1.9)$ \\
\hline Endometrioid & $3(2.8)$ \\
\hline Clear cell & 7 (6.6) \\
\hline Mixed & $1(0.9)$ \\
\hline Adenocarcinoma (NOS) & $7(6.6)$ \\
\hline \multicolumn{2}{|l|}{ FIGO stage, n (\%) } \\
\hline IIB & $1(0.9)$ \\
\hline IIC & $4(3.8)$ \\
\hline IIIA & $3(2.8)$ \\
\hline IIIB & $3(2.8)$ \\
\hline IIIC & $61(57.6)$ \\
\hline IV & $34(32.1)$ \\
\hline \multicolumn{2}{|l|}{ Grade, n (\%) } \\
\hline Poor & $71(67.0)$ \\
\hline Moderate & $23(21.7)$ \\
\hline Well & $5(4.7)$ \\
\hline Not graded (clear cell) & $7(6.6)$ \\
\hline \multicolumn{2}{|c|}{ ECOG performance status, n (\%) } \\
\hline 0 & $86(81.1)$ \\
\hline $1-2$ & $20(18.9)$ \\
\hline \multicolumn{2}{|l|}{ Residual disease, n (\%) (cm) } \\
\hline 0 & $20(18.9)$ \\
\hline $0-2$ & $32(30.2)$ \\
\hline$>2$ & $52(49.1)$ \\
\hline Not specified & $2(1.9)$ \\
\hline
\end{tabular}

hydramine and a histamine $\mathrm{H}_{2}$-receptor antagonist, such as cimetidine. The creatinine clearance was calculated by the method of Cockcroft and Gault (18).

Response evaluation. Clinical response was assessed at the completion of 6 chemotherapy cycles (or after at least 3 completed cycles) via clinical, radiographic and serologic means in accordance with the WHO response criteria (19) and the Rustin criteria (20). Patients with residual disease at start of chemotherapy and who completed at least 3 cycles of chemotherapy $(n=85)$ were evaluable for clinical response evaluation. A complete response (CR) means the disappearance of all target and non-target lesions. A partial response (PR) is determined by at least a $50 \%$ decrease in the product of the longest diameter and the longest perpendicular diameter of target lesions taking as reference the baseline product. Stable disease (SD) signifies neither a sufficient decrease to qualify for partial response nor a sufficient increase to qualify for progressive disease (PD) taking as reference the smallest product of the two diameters since the treatment started. Progression (PD) is at least a $25 \%$ increase in 


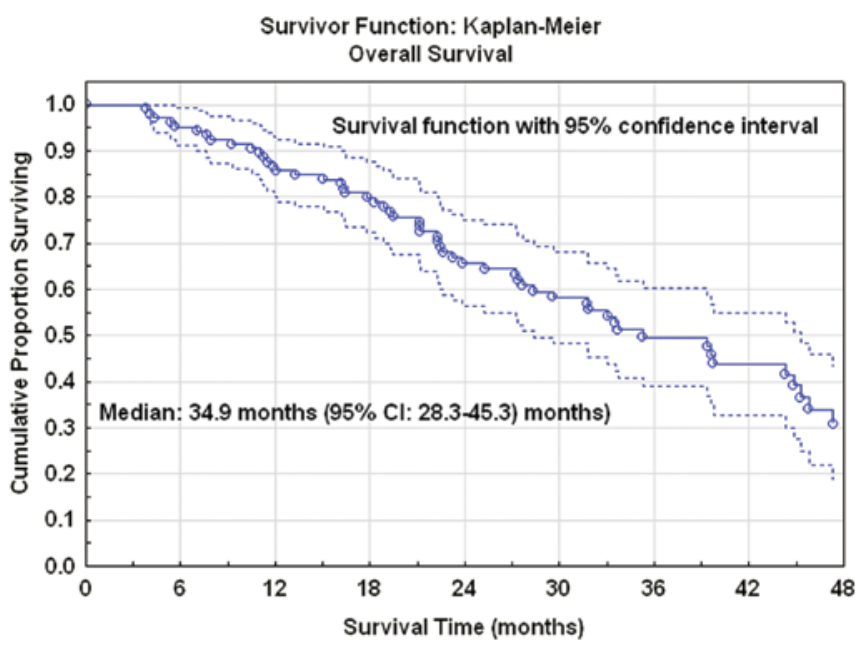

Figure 1. Overall survival of the complete series $(\mathrm{n}=106)$. Kaplan-Meier survival function with $95 \%$ confidence interval.

the product of the diameters of target lesions taking as reference the smallest product recorded since the treatment started or the appearance of one or more new lesions.

Chest and abdominal/pelvic computed tomographic scans were performed before surgery, at baseline before start of chemotherapy, after cycle 3 and after the completion of cycle 6 . If chemotherapy was continued after cycle 6 computed scans were repeated also after cycle 9 . Response evaluation was confirmed after the conclusion of cycle 6 in the determination of the objective response rate. The biochemical marker (CA-125) was evaluated after the last cycle ( $\geq 3$ cycles) of chemotherapy.

Toxicity analysis. Toxicity was graded according to the Common Terminology Criteria for Adverse Events (CTCAE v3.0, 2003) (21). Patients were required to have an absolute neutrophil count $(\mathrm{ANC}) \geq 1.5 \times 10^{9} / 1$ and a platelet count $\geq 100 \times 10^{9} / 1$ on day 1 to receive chemotherapy. Complete blood cell values were obtained weekly until the conclusion of cycle 6 and then subsequently, every 3 weeks. Adequate renal function was defined as serum creatinine $<1.25$ of upper normal limit, and liver function of bilirubin $<$ upper normal limit, AST/ALT $<1.5$ of upper normal limit and ALP $<3$ times of upper normal limit. Symptomatic peripheral neuropathy CTCAE grade $\geq 2$ was an exclusion criterion. All subjects who completed at least 1 cycle of chemotherapy were included in the toxicity analysis. Special attention was paid to the neurological toxicity with clinical tests for 2-point discrimination (the right index finger), the Romberg test, patellar reflex, Achilles reflex and the vibratory sense (the first metatarso-phalangel joint of the right foot). A neurological questionnaire was also filled in by the patients at baseline and at every cycle of chemotherapy.

Quality-of-life measurement. Quality of life was measured at baseline and at every cycle of chemotherapy (cycle 1-9) using the EORTC QLQ-C30 (version 3.0) and the QLQ-OV28 instruments. The compliance was high and $93-99 \%$ of the patients completed the questionnaires. The results were analyzed and presented according to the EORTC guidelines (22) for these two questionnaires.

Evaluation. Progression-free survival (PFS) was defined as the date of diagnosis to the first observation of disease progression or death due to any cause. Overall survival was defined as time from diagnosis to the date of death due to any cause. If a subject had not progressed or died, PFS was censored at the time of last follow-up. The median follow-up of all patients alive was 33 months (1-60 months).

Statistical analyses. The overall response rate (ORR) for the combination docetaxel-carboplatin given as a 3-weekly combination is reported to be $65 \%$ (9). The alternative hypothesis is that the ORR in the weekly docetaxel - 3-weekly carboplatin regimen is $\leq 50 \%$. This non-randomized phase II study with a one sample, single stage design has been dimensioned to detect this difference in ORR with a significance level of 5\% and a power of $80 \%$. With 85 patients these criteria will be met (95\% CI 55-74\%). To compensate for patients included and not evaluable for response 110 patients were included. One hundred and six patients were correctly included according to inclusion and exclusion criteria. Survival analyses (PFS and OS) were done by the Kaplan-Meier method and $95 \%$ confidence intervals (CI) were presented. Median progression-free and overall survival was also calculated. General regression models (GRM) were used in analyses of the life quality data. The Statistica software (StatSoft, Inc., Tulsa, OK, USA) version 10.0 was used for all statistical analyses in this study.

\section{Results}

Response rate. Thirty-eight patients demonstrated a clinical complete response $(44.7 \%)$ and 29 patients exhibited a partial response $(34.1 \%)$, resulting in a total clinical response rate of $78.8 \%$ (95\% CI 70.1-87.5\%). Sixteen patients had stable disease $(18.8 \%)$ and 2 patients developed progressive disease $(2.4 \%)$. Twenty-one patients were not evaluable for tumor response $(<3$ courses of chemotherapy or no measurable disease). Seventyseven patients showed a biochemical (CA-125) response (92.8\%) (95\% CI 87.2-98.4\%), 5 patients stable disease (6.0\%), and one patient had tumor progression. Twenty-three patients were not evaluable biochemically (CA-125).

Survival rates. The median overall survival rate was 35.3 months and progression-free survival rate 12.0 months (Fig. 1). The 3-year overall survival rate was $49.6 \%$ (95\% CI 38.8$60.4 \%$ ) and the progression-free survival rate $16.0 \%$ (95\% CI 8.2-23.8\%) (Table II).

Toxicity. In 36 patients (34.0\%) grade 3-4 neutropenia were recorded. Grade 1-2 neutropenia were recorded in 30 patients (28.3\%). Febrile neutropenia was recorded in 8 patients $(7.6 \%)$ and septicemia in one patient. Grade 3-4 thrombocytopenia was recorded in only 6 patients $(5.7 \%)$. None of the patients exhibited grade 3-4 anemia. Three patients were withdrawn from the study due to hematological toxicity. Table III illustrates the hematological toxicity.

Fatigue was the most frequently $(53.8 \%)$ recorded nonhematological side effect associated with this regimen. The 
Table II. Progression-free and overall survival data.

\begin{tabular}{lcccc}
\hline & $\begin{array}{c}\text { Progression-free } \\
\text { survival rate (PFS) }\end{array}$ & $\begin{array}{c}\text { 95\% Confidence } \\
\text { interval }\end{array}$ & $\begin{array}{c}\text { Overall survival } \\
\text { rate (OS) }\end{array}$ & $\begin{array}{c}95 \% \text { Confidence } \\
\text { interval }\end{array}$ \\
\hline 1 year & 51.4 & $80.2-93.1$ & 86.7 & $80.2-93.1$ \\
2 year & 23.1 & $14.7-31.4$ & 65.7 & $56.2-75.1$ \\
3 year & 16.0 & $8.2-23.8$ & 49.6 & $38.8-60.3$ \\
4 year & 11.4 & $3.5-19.3$ & 30.9 & $18.4-43.4$ \\
\hline
\end{tabular}

Table III. Hematological toxicity in the complete series $(n=106)$.

\begin{tabular}{lccr}
\hline & Neutropenia & $\begin{array}{c}\text { Thrombocytopenia } \\
\text { no. of patients }(\%)\end{array}$ & Anemia \\
\hline Grade 1 & $42(39.6)$ & $55(51.9)$ & $56(52.8)$ \\
Grade 2 & $23(21.7)$ & $10(9.4)$ & $50(47.2)$ \\
Grade 3 & $30(28.3)$ & $5(4.7)$ & $0(0.0)$ \\
Grade 4 & $6(5.7)$ & $1(0.9)$ & $0(0.0)$ \\
\hline
\end{tabular}

second most common adverse event was problems with watery eyes and tearing (epiphora) affecting 53 patients (50\%). Nail changes were rather common with this weekly schedule and 19 grade 1, 12 grade 2 and 1 grade 3 cases were recorded. Alopecia grade 1-2 was noted in $58(54.7 \%)$ cases and edema grade 1-3 in $24(22.6 \%)$ patients. Table IV illustrates various types of non-hematological toxicity.

Neurotoxicity - symptoms and signs. Forty-five (41.5\%) patients developed grade 1 sensory neuropathy and 7 patients $(6.6 \%)$ manifested grade 2 sensory neuropathy, but no patients exhibited grade 3 or higher sensory neuropathy. Twelve (11.3\%) patients developed grade 1 motor neuropathy and $2(1.9 \%)$ patients grade 2 motor neuropathy.

Formalized neurological tests were performed at baseline and after 3 and 6 cycles of chemotherapy (Table V). Two-point discrimination (tip of index finger) and Romberg test were normal $(100 \%)$ after 6 courses of treatment. Patellar reflex and vibratory sense were normal in $\sim 80 \%$ before, during and after chemotherapy. The only significant change was the presence of a normal Achilles reflex which was $80 \%$ at baseline and $65 \%$ after chemotherapy.

Table IV. Non-hematological toxicity of the complete series $(n=106)$.

\begin{tabular}{|c|c|c|c|c|}
\hline \multirow[b]{2}{*}{ Type of toxicity } & \multicolumn{4}{|c|}{ No. of patients (\%) } \\
\hline & Grade 1 & Grade 2 & Grade 3 & Grade 4 \\
\hline Neuropathy - sensory & $45(42.4)$ & 7 (6.6) & $1(0.9)$ & $0(0.0)$ \\
\hline Neuropathy - motor & $12(11.3)$ & $2(1.9)$ & $0(0.0)$ & $0(0.0)$ \\
\hline Edema & $17(16.0)$ & $6(5.7)$ & $1(0.9)$ & $0(0.0)$ \\
\hline Mucositis & $23(21.7)$ & $5(4.7)$ & $6(5.7)$ & $0(0.0)$ \\
\hline Nail changes & $19(17.9)$ & $12(11.3)$ & $1(0.9)$ & $0(0.0)$ \\
\hline Alopecia & $28(26.4)$ & $30(28.3)$ & $0(0.0)$ & $0(0.0)$ \\
\hline Nausea & $44(41.5)$ & $16(15.1)$ & $9(8.5)$ & $0(0.0)$ \\
\hline Vomiting & $16(15.1)$ & $16(15.1)$ & $6(5.7)$ & $1(0.9)$ \\
\hline Diarrhea & $38(35.9)$ & $12(11.3)$ & $5(4.7)$ & $0(0.0)$ \\
\hline Constipation & $33(31.1)$ & $14(13.2)$ & $0(0.0)$ & $1(0.9)$ \\
\hline Other types of toxicity & \multicolumn{4}{|c|}{ All grades included } \\
\hline Fatigue & \multicolumn{4}{|c|}{$57(53.8)$} \\
\hline Tearing eyes & \multicolumn{4}{|c|}{$53(50.0)$} \\
\hline Taste disturbances & \multicolumn{4}{|c|}{$50(47.2)$} \\
\hline Rash & \multicolumn{4}{|c|}{$15(14.2)$} \\
\hline Dizziness & \multicolumn{4}{|c|}{$12(11.3)$} \\
\hline Hoarseness & \multicolumn{4}{|c|}{$9(8.5)$} \\
\hline Heartburn/gastritis & \multicolumn{4}{|c|}{9 (8.5) } \\
\hline
\end{tabular}


Table V. Neurological tests $(n=106)$.

\begin{tabular}{lccc}
\hline & \multicolumn{3}{c}{ Normal (\%) } \\
\cline { 2 - 4 } Type of test & Baseline & Cycle 3 & Cycle 6 \\
\hline Two-point discrimination & & & \\
Romberg test & 98 & 100 & 100 \\
Patellar reflex & 99 & 98 & 100 \\
Achilles reflex $^{\text {Vibratory sense }}$ & 81 & 86 & 78 \\
& 80 & 85 & 65 \\
\hline
\end{tabular}

${ }^{\mathrm{a} T i p}$ of right index finger; ${ }^{\mathrm{b}}$ First metatarso-phalangeal joint of left foot, $128 \mathrm{~Hz}$.

A neurological questionnaire was also filled in by the patients at baseline and after 3 and 6 courses of chemotherapy (Table VI). All signs and symptoms of peripheral neuropathy of the feet and fingers increased substantially from baseline and during chemotherapy. Tingling or numbness of the feet was most frequent and increased from 2.9 to $45 \%$ and for the fingers to $33 \%$. Problems with pain, discomfort and weakness were less frequent but still significant clinical problems. Neurological symptoms were normally $(65-83 \%)$ located at both sides but increased in frequency from occasionally to constantly during treatment. Problems and restrictions of daily activities, problems to handle small objects and medication needed for the neurological symptoms increased during treatment but were most pronounced after cycle 6 . However, the majority of the patients (60\%) graded their symptoms as 'bearable' and only 7\% as 'very troublesome' or 'unbearable'.

Quality-of-life. The global quality of life scores (functional scale) according to EORTC QLQ-C30 increased from 58.8 at baseline (post-operative value) to 64.4 after the first course of chemotherapy and then slowly decreased again, course by course, to 50.3 after 6 completed cycles (Fig. 2). This decrease of 14.1 points was of moderate degree and probably associated with the burden of the chemotherapy treatment. The last cycle (number 6) seems to lower the score more than the preceding cycles (number 2-5) (Table VII). The physical score was stable from baseline (77.2) to cycle 3 (77.3), but then slowly decreased to 71.9 after 6 completed cycles. This difference is of small clinical relevance. The role and emotional scales showed a rise after course one, but were then rather stable throughout the treatment. The cognitive score was stable during all cycles, and the social score rose after the first two cycles and then fell back after six courses of chemotherapy.

Scores from the symptoms and side effects scales showed for fatigue, nausea and vomiting, dyspnea, and appetite loss a similar pattern with increased score values during cycles 5 and 6 but rather stable values from base line and during cycles 1-4. Pain was reduced from 24.5 to 16.8 when chemotherapy started and then remained stable during all courses. The problem with insomnia was unchanged during chemotherapy. After the first course of chemotherapy the problem with constipation successively decreased to 8.3 after 6 courses. On the other hand, diarrhea was more frequent during chemotherapy than at
Table VI. Neurological questionnaire $(n=106)$.

\begin{tabular}{lccc}
\hline Questions & Baseline & Cycle 3 & Cycle 6 \\
\hline $\begin{array}{l}\text { Tingling or numbness of } \\
\text { the feet, Yes (\%) }\end{array}$ & 2.9 & 21 & 45 \\
$\begin{array}{l}\text { Tingling or numbness of } \\
\text { the fingers, Yes (\%) }\end{array}$ & 2.9 & 16 & 33 \\
$\begin{array}{l}\text { Pain or discomfort of } \\
\text { the feet, Yes (\%) }\end{array}$ & 3.8 & 8 & 20 \\
$\begin{array}{l}\text { Pain or discomfort of } \\
\text { the fingers, Yes (\%) }\end{array}$ & 1.9 & 9 & 12 \\
$\begin{array}{l}\text { Weakness of the feet, Yes (\%) } \\
\text { Weakness of the fingers, Yes (\%) }\end{array}$ & 1.9 & 11 & 22 \\
$\begin{array}{l}\text { Localization of the neurological } \\
\text { symptoms }\end{array}$ & & 11 & 25 \\
$\quad \begin{array}{l}\text { Right side (\%) } \\
\text { Left side (\%) } \\
\text { Both sides (\%) }\end{array}$ & 17 & 19 & 13 \\
\end{tabular}

Frequency of the neurological symptoms

$\begin{array}{llll}\text { Constantly (\%) } & 17 & 26 & 40 \\ \text { Occasionally (\%) } & 83 & 74 & 60\end{array}$

Problems with insomnia due to neurological symptoms

$\begin{array}{cccc}\text { Yes }(\%) & 50 & 16 & 17 \\ \text { No }(\%) & 50 & 84 & 83\end{array}$

Problems to handle small objects

(coins, buttons)

$\begin{array}{llll}\text { Yes }(\%) & 14 & 10 & 26 \\ \text { No }(\%) & 86 & 90 & 74\end{array}$

Restriction of daily activities due to neurological symptoms

$\begin{array}{llll}\text { Yes }(\%) & 17 & 26 & 35 \\ \text { No }(\%) & 83 & 74 & 65\end{array}$

Medication needed for the

neurological symptoms

$\begin{array}{lrrr}\text { Yes }(\%) & 0 & 4 & 17 \\ \text { No }(\%) & 100 & 96 & 83\end{array}$

Intensity grading of the symptoms

$\begin{array}{lrrr}\text { Unbearable }(\%) & 0 & 3 & 0 \\ \text { Very troublesome (\%) } & 0 & 6 & 7 \\ \text { Troublesome }(\%) & 17 & 23 & 20 \\ \text { A bit troublesome }(\%) & 33 & 13 & 13 \\ \text { Bearable }(\%) & 50 & 55 & 60\end{array}$

baseline. The impact on the financial situation increased from 8.4 at baseline to 14.9 at completion of chemotherapy, which was a minor impact.

Quality-of-life scores were also measured by the EORTC QLQ-OV28 scale which is specific for ovarian carcinoma (Table VIII). Abdominal and gastrointestinal side effects were 
Table VII. Quality-of-life scores (mean, SD) according to QLQ-C30 (n=104).

\begin{tabular}{|c|c|c|c|c|c|c|c|}
\hline Type of area & Baseline & Cycle 1 & Cycle 2 & Cycle 3 & Cycle 4 & Cycle 5 & Cycle 6 \\
\hline \multicolumn{8}{|l|}{ Functional scales } \\
\hline Physical (items 1-5) & $77.2(18)$ & $77.8(18)$ & $78.1(18)$ & $77.3(21)$ & 74.4 (19) & $73.4(19)$ & $71.9(19)$ \\
\hline Role (items 6-7) & $50.3(36)$ & $65.2(32)$ & $61.3(33)$ & $63.7(34)$ & $61.1(32)$ & $58.0(33)$ & $57.0(33)$ \\
\hline Emotional (items 21-24) & $72.9(21)$ & $81.8(22)$ & $82.2(20)$ & $80.0(20)$ & $80.5(16)$ & $76.4(20)$ & $74.8(22)$ \\
\hline Cognitive (items 20, 25) & $87.1(16)$ & $90.0(17)$ & $88.7(16)$ & $89.4(15)$ & $87.7(15)$ & $86.6(17)$ & $84.5(18)$ \\
\hline Social (items 26-27) & $70.5(25)$ & $81.0(23)$ & $79.6(23)$ & $76.4(27)$ & $75.8(22)$ & $74.4(25)$ & $70.5(25)$ \\
\hline Global (items 29-39) & $58.8(22)$ & $64.4(22)$ & $62.2(20)$ & $61.1(19)$ & $58.1(19)$ & $57.2(19)$ & $50.3(24)$ \\
\hline \multicolumn{8}{|l|}{$\begin{array}{l}\text { Symptoms and side effects } \\
\text { scales }\end{array}$} \\
\hline Fatigue (items $10,12,18$ ) & $36.9(24)$ & $41.4(24)$ & $37.3(24)$ & $39.2(26)$ & $41.1(21)$ & $44.3(23)$ & $46.1(23)$ \\
\hline Nausea and vomit (items 14-15) & $7.4(17)$ & $12.6(19)$ & $11.2(17)$ & $10.6(17)$ & $9.1(16)$ & $12.6(20)$ & $22.9(19)$ \\
\hline Pain (items 9, 19) & $24.5(24)$ & $16.8(22)$ & $13.9(20)$ & $12.3(20)$ & $10.0(18)$ & $17.1(24)$ & $16.1(22)$ \\
\hline Dyspnea (item 8) & $18.2(28)$ & $18.5(26)$ & $21.3(28)$ & $22.6(29)$ & $27.3(27)$ & $29.6(31)$ & $34.5(30)$ \\
\hline Insomnia (item 11) & $32.1(32)$ & $29.7(32)$ & $28.6(27)$ & $26.7(27)$ & $24.2(25)$ & $25.8(27)$ & $24.4(30)$ \\
\hline Appetite loss (item 13) & $20.4(30)$ & $20.8(31)$ & $22.0(34)$ & $22.2(32)$ & $21.9(32)$ & $31.0(36)$ & $25.3(30)$ \\
\hline Constipation (item 16) & $15.7(26)$ & $19.4(29)$ & $12.4(21)$ & $13.0(22)$ & $13.0(25)$ & $10.5(21)$ & $8.3(21)$ \\
\hline Diarrhea (item 17) & $8.5(22)$ & $21.7(32)$ & $12.0(21)$ & $12.6(23)$ & $12.6(21)$ & $12.6(21)$ & $15.8(26)$ \\
\hline Financial impact (item 28) & $8.4(19)$ & $10.1(20)$ & $10.8(22)$ & $12.8(23)$ & $14.5(24)$ & $13.6(23)$ & $14.9(22)$ \\
\hline
\end{tabular}

Table VIII. Quality-of-life scores (mean, SD) according to QLQ-OV28 ( $\mathrm{n=104}$ ).

\begin{tabular}{lccccccc}
\hline Type of area & Baseline & Cycle 1 & Cycle 2 & Cycle 3 & Cycle 4 & Cycle 5 & Cycle 6 \\
\hline $\begin{array}{l}\text { Symptoms and side } \\
\text { effects scales }\end{array}$ & & & & & & & \\
$\begin{array}{l}\text { Abdominal/GI } \\
\text { (items 1-6) }\end{array}$ & $26.1(20)$ & $21.7(17)$ & $20.1(16)$ & $19.2(17)$ & $17.6(15)$ & $18.7(14)$ & $20.8(17)$ \\
$\begin{array}{l}\text { Peripheral neuropathy } \\
\text { (items 11-12) }\end{array}$ & $1.3(6)$ & $3.7(8)$ & $8.5(17)$ & $9.7(18)$ & $11.5(19)$ & $15.0(23)$ & $23.5(27)$ \\
$\begin{array}{l}\text { Hormonal } \\
\text { (items 18-19) }\end{array}$ & $19.0(21)$ & $20.7(24)$ & $18.1(24)$ & $15.0(21)$ & $13.1(23)$ & $12.0(19)$ & $11.3(21)$ \\
$\begin{array}{l}\text { Body image } \\
\text { (items 20-21) }\end{array}$ & $24.7(27)$ & $19.6(20)$ & $21.7(23)$ & $24.1(26)$ & $28.5(29)$ & $29.5(28)$ & $34.8(30)$ \\
$\begin{array}{l}\text { Attitude to disease } \\
\text { (items 22-24) }\end{array}$ & $47.0(24)$ & $41.2(23)$ & $41.0(25)$ & $43.3(27)$ & $42.5(25)$ & $40.8(25)$ & $47.7(29)$ \\
$\begin{array}{l}\text { Chemo side effects } \\
\text { (items 13-17) }\end{array}$ & $11.4(11)$ & $13.5(13)$ & $16.8(15)$ & $15.5(14)$ & $16.1(13)$ & $17.9(13)$ & $20.7(14)$ \\
$\begin{array}{l}\text { Other single items } \\
\text { (items 7-10) }\end{array}$ & $25.0(8)$ & $31.3(17)$ & $44.0(23)$ & $45.3(17)$ & $40.5(15)$ & $43.8(16)$ & $43.2(18)$ \\
$\begin{array}{l}\text { Sexuality } \\
\text { (items 25-28) }\end{array}$ & $61.7(11)$ & $66.2(10)$ & $65.6(11)$ & $67.9(7)$ & $69.4(10)$ & $64.3(12)$ & $68.3(15)$ \\
\hline
\end{tabular}

reduced from 26.1 at baseline to 20.8 when chemotherapy was finished and stable during courses 1-6. Hormonal symptoms showed a similar pattern with successive improvement. Body image and attitude to disease were unchanged from baseline and during treatment as was sexuality. Significant increases were noted for peripheral neuropathy from 1.3 at baseline to
23.5 at completion of the chemotherapy (Fig. 3) and for other chemo side effects from 11.4 to 20.7 .

The global quality of life (QLQ-C30) was significantly associated with gastrointestinal side effects and the attitude towards disease at baseline and after the first course of chemotherapy, but changed during treatment to neurological 


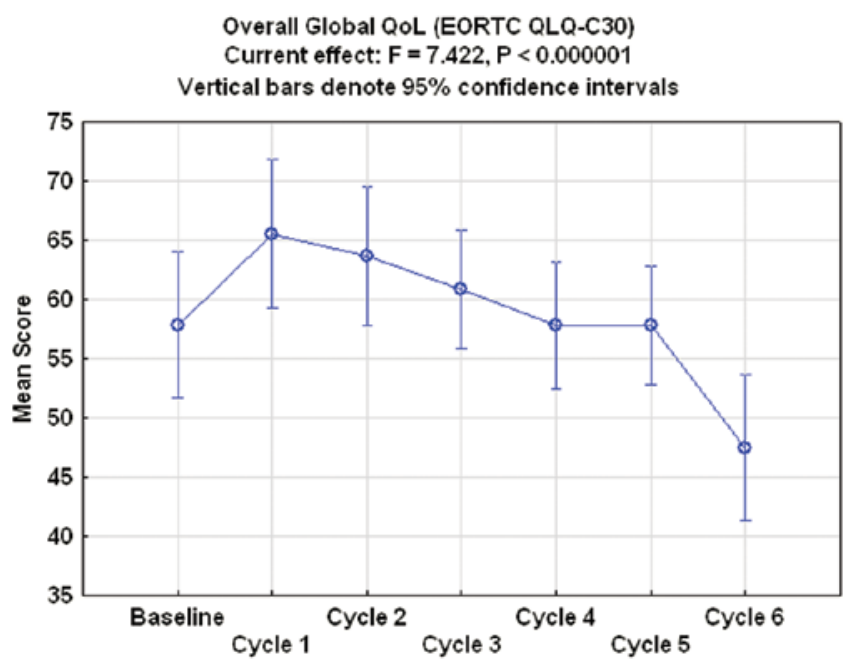

Figure 2. Overall, global quality-of-life measured on EORTC QLQ-C30 scale. Mean score and $95 \%$ confidence interval at baseline and during 6 courses of chemotherapy.

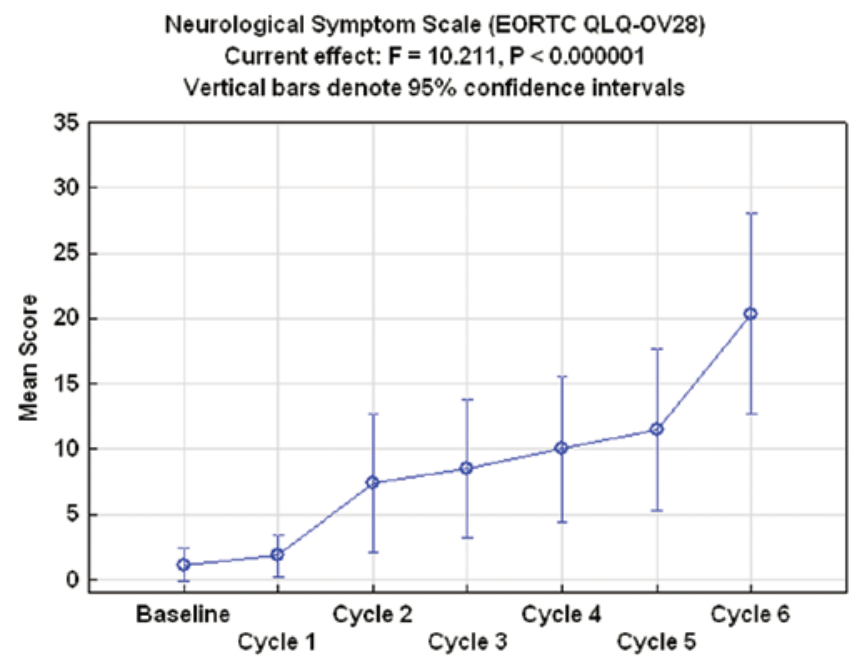

Figure 3. Neurological symptoms measured on EORTC QLQ-OV28 scale. Mean score and 95\% confidence interval at baseline and during 6 courses of chemotherapy.

side effects and other side effects related to chemotherapy in addition to gastrointestinal side effects all measured on the QLQ-OV28 scale (Fig. 4). The physical score on the QLQ-C30 scale was associated with gastrointestinal side effects and attitude to disease at baseline and chemotherapy-related side effects after start of chemotherapy.

\section{Discussion}

In an attempt to improve the efficacy and tolerability of standard 3-week regimens of the combination of carboplatin and a taxane, the activity of weekly administration of docetaxel and 3-weekly carboplatin was studied in this prospective, multicenter phase II trial. This has not been done before in a 1-line setting in primary treatment of advanced ovarian cancer. In a Japanese study, Katsumata et al (6) investigated the efficacy of

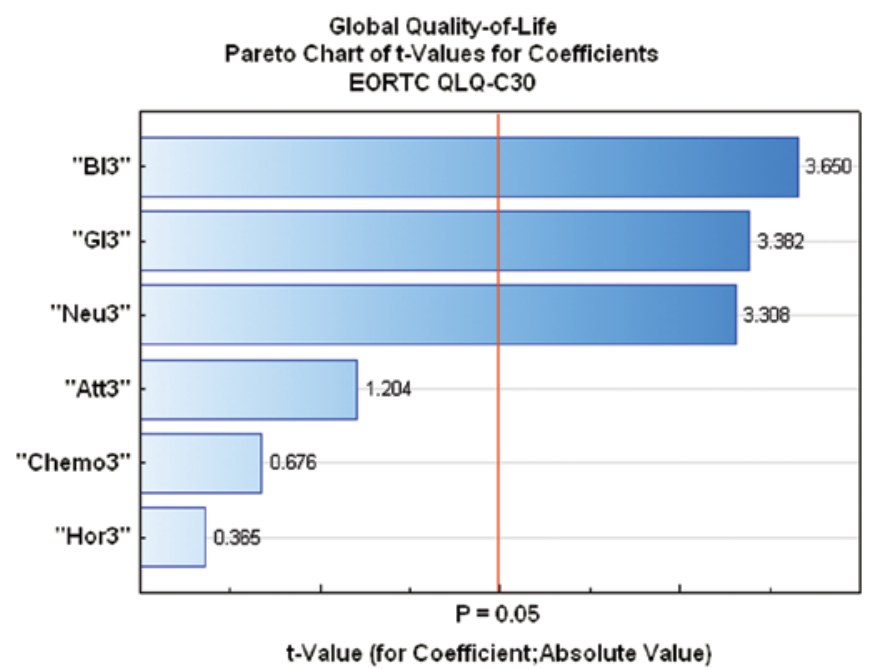

Figure 4. Pareto chart of t-values for coefficients of six variables from the EORTC QLQ-OV28 scale and the global quality of life (QoL) measured by the QLQ-C30 scale after 3 courses of chemotherapy. The body image (BI3), gastrointestinal side effects (GI3) and the neurological side effects (Neu3) were statistically significantly associated with global QoL. Attitude towards disease (Att3), other side effects of chemotherapy (Chemo3) and hormonal symptoms (Hor3) were non-significant variables.

the combination of weekly dose dense paclitaxel $\left(80 \mathrm{mg} / \mathrm{m}^{2}\right)$ and carboplatin $(\mathrm{AUC}=6)$ on Days 1,8 and 15 compared with every 3 weeks of paclitaxel $\left(180 \mathrm{mg} / \mathrm{m}^{2}\right)$ and carboplatin $(\mathrm{AUC}=6)$ in patients with previously untreated ovarian cancer. Although the two regimens had relatively similar toxicity, progressionfree survival was significantly improved in the patients who received the dose dense regimen (median 28 vs. 17 months; $\mathrm{P}=0.0014)$. Data of weekly chemotherapy are reported in a number of previous studies $(4,5)$. We encountered an overall response rate of $78.8 \%$, which is superior to the $55.8 \%$ reported by Katsumata et al (8) but lower than the $92 \%$ reported by Safra et al (13) who used weekly carboplatin $(\mathrm{AUC}=2)$ and paclitaxel $\left(80 \mathrm{mg} / \mathrm{m}^{2}\right)$ on Days 1,8 and 15 of a 28 -day cycle for primary treatment of advanced ovarian carcinoma. Micha et al (23) and Penson et al (24) have reported response rates of 80 and $76 \%$, respectively, when adding bevacizumab to paclitaxel and carboplatin in treatment of advanced-stage ovarian cancer. As in these studies we have also used the Word Health Organization criteria (19) for clinical response evaluation. However, the median progression-free survival of 12 months in our study was substantially lower than the 26 months reported by Safra et al (4) using weekly carboplatin and paclitaxel in treatment of advanced ovarian carcinoma. In our series the majority of the patients had stage III-IV disease $(90 \%)$ with suboptimal surgery $(80 \%)$.

In terms of hematological toxicity, grade 3-4 neutropenia were recorded in $34 \%$ of the patients during any of the six courses administered. Sehouli et al (5) reported that $28 \%$ of their patients with advanced ovarian cancer developed grade 3-4 neutropenia after weekly paclitaxel $\left(100 \mathrm{mg} / \mathrm{m}^{2}\right)$ and carboplatin $(\mathrm{AUC}=2)$. Thrombocytopenia was infrequent and grade 3-4 were recorded in only $6 \%$ of the patients. None of the patients experienced grade 3-4 anemia. Thus, the hematological toxicity was quite manageable and not a 
clinical problem. However, in 7 patients hematological toxicity (neutropenia) was given as the reason for not completing 6 courses of chemotherapy. Colony-stimulating factors were normally not used with this regimen.

In the present study, sensory neuropathy grades 1 and 2 developed in 44 and 7 patients, respectively; none exhibited severe neurotoxicity grade 3 . These results are comparable to Micha et al (23) and Sehouli et al (5) who reported low rates of severe peripheral neuropathy ( $2.3 \%$ grade 3$)$. One would suspect that lower, weekly doses of taxanes would mitigate toxicity (4). However, Seidman et al (25) reported that neurotoxicity was a dose-limiting factor after weekly paclitaxel $\left(80 \mathrm{mg} / \mathrm{m}^{2}\right)$ for the treatment of metastatic breast cancer. Thus, docetaxel seems to have a lower rate of disabling neurotoxicity than paclitaxel when given on a weekly schedule. The weekly schedule also seems superior to 3-week schedules of both paclitaxel and docetaxel with regard to both sensory and motor neurotoxicity.

Epiphora was another type of toxicity recorded in 50\% of the patients and seems to be specifically associated with weekly administration of docetaxel. Esmaeli et al (26) from MD Anderson Cancer Center reported on 148 patients with this type of side effect. Thirty of 71 patients given weekly docetaxel needed surgery to correct epiphora. Of the patients who received docetaxel every 2 or 3 weeks, only 3 required a surgical intervention to correct epiphora. A schedule of docetaxel given every 2 weeks has also been tested with favorable outcome and toxicity profile and should be further evaluated in larger series of advanced ovarian cancer patients (27).

This study also included quality of life analyses using the EORTC QLQ-C30 and QLQ-OV28 instruments. The rate of drop-out of patients, despite a well-known phenomenon (28), was low $(<10 \%)$ in this trial. Global quality of life increased from baseline and during the first part of chemotherapy and then gradually decreased again due to chemotherapy-induced side effects (Fig. 2). Gastrointestinal side effects and neurological side effects and also other types of chemotherapy-related side effects were most significantly associated with the global quality-of-life score. The results of our life quality analyses are similar to the results from studies using the standard carboplatin-paclitaxel regimen administered every three weeks and superior of the cisplatin-paclitaxel regimen (29). A reference standard population (1,139 German women) (30) showed superior quality-of-life scores for all items measured on the EORTC QLQ-C30 scale.

Peripheral neuropathy is one the most important side effects of platinum and taxane chemotherapy, both are standard drugs for treatment of ovarian cancer. These side effects interfere with self-care activities, physical and role activities, and quality of life (31).

One-third of patients undergoing cisplatin and paclitaxel experienced long-term toxicity, such as numbness and tingling (32). This limits the use of paclitaxel in second-line therapy despite improved survival compared with a platinum agent alone (33). In this study neurological tests were performed, specific neurological questionnaires were filled in by the patients and the EORTC QLQ-OV28 scale was used as a complement to EORTC QLQ-C30. The peripheral neurological side effects increased substantially during the courses of chemotherapy, and especially during courses 5 and 6 (Fig. 3). The overall quality of life was significantly influenced by this side effect. All neurological tests were normal, except for the Achilles reflex that was weak or absent in $35 \%$ of the cases at the end of the chemotherapy compared to $20 \%$ at baseline. Tingling and numbness of the feet (45\%) and fingers (33\%) were the most common type of discomfort during and after treatment. Weakness of hands or feet was less common, but occurred in 25 and $22 \%$, respectively. The neurological side effects were normally bilateral, constantly present in $40 \%$, restricted daily activities in $35 \%$, but were graded as a bit troublesome or bearable in the majority of the cases (73\%). Despite these figures peripheral neuropathy is a minor problem for docetaxel compared with paclitaxel regimens in treatment of ovarian cancer. This is the principal advantage of this taxane.

Docetaxel is still an alternative to paclitaxel both in firstline and second-line chemotherapy regimens for advanced ovarian carcinoma. Dose-dense schedules with weekly or twice weekly administrations of the drug are also of interest to further explore to improve and optimize the efficacy and the toxicity profile of docetaxel chemotherapy combinations.

\section{Acknowledgements}

The authors thank Sanofi-Aventis AB, Bromma, Sweden, for the financial support by grants.

\section{References}

1. Ferlay J, Shin HR, Bray F, et al: GLOBOCAN 2008, Cancer incidence and mortality worldwide: IARC CaseBase No. 10. International Agency for Research on Cancer, Lyon, 2010.

2. McGuire WP, Hoskins WJ, Brady MF, et al: Cyclophosphamide and cisplatin compared with paclitaxel and cisplatin in patients with stage III and stage IV ovarian cancer. N Engl J Med 334: $1-6,1996$.

3. Copeland LJ, Bookman M and Trimble E; Gynecologic Oncology Group Protocol GOG 182-ICON5: Clinical trials of newer regimens for treating ovarian cancer: the rationale for Gynecologic Oncology Group Protocol GOG 182-ICON5 Gynecol Oncol 90: S1-S7, 2003.

4. Safra T, Menczer J, Bernstein RM, et al: Combined weekly carboplatin and paclitaxel as primary treatment of advanced epithelial ovarian carcinoma. Gynecol Oncol 114: 215-218, 2009.

5. Sehouli J, Stengel D, Mustea A, et al: Weekly paclitaxel and carboplatin (PC-W) for patients with primary advanced ovarian cancer: results of a multicenter phase-II study of the NOGGO. Cancer Chemother Pharmacol 61: 243-250, 2008.

6. Katsumata N, Yasuda M, Takahashi F, et al: Dose-dense paclitaxel once a week in combination with carboplatin every 3 weeks for advanced ovarian cancer: a phase 3 , open-label, randomised controlled trial. Lancet 374: 1331-1338, 2009.

7. Kavanagh JJ: Docetaxel in the treatment of ovarian cancer. Oncology 16: 73-81, 2002.

8. Katsumata N: Docetaxel: an alternative taxane in ovarian cancer. Br J Cancer 89: S9-S15, 2003.

9. Vasey PA, Jayson GC, Gordon A, et al: Phase III randomized trial of docetaxel-carboplatin versus paclitaxel-carboplatin as first-line chemotherapy for ovarian carcinoma. J Natl Cancer Inst 96: 1682-1691, 2004.

10. Kushner DM, Connor JP, Sanchez F, et al: Weekly docetaxel and carboplatin for recurrent ovarian and peritoneal cancer: a phase II trial. Gynecol Oncol 105: 358-364, 2007.

11. Tinker AV, Gebski V, Fitzharris B, et al: Phase II trial of weekly docetaxel for patients with relapsed ovarian cancer who have previously received paclitaxel - ANZGOG 02-01. Gynecol Oncol 104: 647-653, 2007.

12. Gupta D, Owers RL, Kim M, et al: A phase II study of weekly topotecan and docetaxel in heavily treated patients with recurrent uterine and ovarian cancers. Gynecol Oncol 113: 327-330, 2009. 
13. Safra T, Bernstein Molho R, Menzcher J, et al: A feasibility study of weekly docetaxel with capecitabine in ovarian cancer: a promising combination of two active drugs with a potential for synergism. Chemotherapy 55: 298-302, 2009.

14. Terauchi F, Hirano T, Taoka H, et al: Weekly docetaxel for patients with platinum/paclitaxel/irinotecan-resistent relapsed ovarian cancer: a phase I study. Int J Clin Oncol 8: 348-351, 2003.

15. Berkenblit A, Seiden MV, Matulonis UA, et al: A phase II trial of weekly docetaxel in patients with platinum-resitent epithelial ovarian, primary peritoneal serous cancer, or fallopian tube cancer. Gynecol Oncol 95: 624-631, 2004.

16. Komiyama S, Tsuji H, Asai S, et al: A pilot study of weekly docetaxel therapy for recurrent ovarian cancer, tubal cancer, and primary peritoneal cancer. Eur J Gynaecol Oncol 6: 299-302, 2005.

17. Calvert AH, Newell DR, Gunbrell LA, et al: Carboplatin dosage: prospective evaluation of a simple formula based on renal function. J Clin Oncol 7: 1748-1756, 1989.

18. Cockcroft DW and Gault MH: Prediction of creatinine clearance from serum creatinine. Nephron 16: 31-41, 1976.

19. Green S and Weiss GR: Southwest oncology group standard response criteria, endpoint definitions and toxicity criteria. Invest New Drugs 10: 239-253, 1992.

20. Rustin GJ, Marples M and Nelstrop AE: Use of CA-125 in clinical trial evaluation of new therapeutic drugs for ovarian cancer. Clin Cancer Res 10: 3919-3926, 2004.

21. National Institute of Health: Common Terminology Criteria for Adverse Events (Version 3.0). National Institute of Health, Washington, DC, 2003.

22. Fayers P, Aaronson N, Bjordal K, et al: EORTC QLQ-C30 Scoring Manual. 3rd edition. EORTC Publications, Brussels, 2001.

23. Micha JP, Goldstein BH, Rettenmaier MA, et al: A phase II study of outpatient first-line paclitaxel, carboplatin, and bevacizumab for advanced-stage epithelial ovarian, peritoneal, and fallopian tube cancer. Int J Gynecol Cancer 17: 771-776, 2007.

24. Penson RT, Dizon DS, Cannistra SA, et al: Phase II study of carboplatin, paclitaxel, and bevacizumab with maintenance bevacizumab as first-line chemotherapy for advanced mullerian tumors. J Clin Oncol 28: 154-159, 2010.
25. Seidman AD, Berry D, Cirrincione C, et al: Randomized phase III trial of weekly compared with every-3-weeks paclitaxel for metastatic breast cancer, with trastuzumab for all HER-2 overexpressors and random assignment to trastuzumab or not in HER-2 non-overexpressors: final results of Cancer and Leukemia Group B protocol 9840. J Clin Oncol 26: 1642-1649, 2008.

26. Esmaeli B, Hidaji L, Adinin RB, et al: Blockage of the lacrimal drainage apparatus as a side effect of docetaxel therapy. Cancer 98: 504-507, 2003

27. Oishi T, Kigawa J, Fujiwara K, et al: A feasibility study on biweekly administration of docetaxel for patients with recurrent ovarian cancer. Gynecol Oncol 90: 421-424, 2003.

28. Bezjak A, Tu D, Bacon M, et al: Quality of life in ovarian cancer patients: comparison of paclitaxel plus cisplatin, with cyclophosphamide plus cisplatin in randomized study. J Clin Oncol 22: 4595-4603, 2004.

29. Greimel ER, Bjelic-Radisic V, Pfisterer J, et al: Randomized study of the Arbeitsgemeinschaft Gynaekologische Onkologie Ovarian Cancer Study Group comparing quality of life in patients with ovarian cancer treated with cisplatin/paclitaxel versus carboplatin/paclitaxel. J Clin Oncol 24: 579-586, 2006.

30. Schwarz R and Hinz A: Reference data for the quality of life questionnaire EORTC QLQ-C30 in the general German population. Eur J Cancer 37: 1345-1351, 2001.

31. Almadrones L, McGuire DB, Walczak JR, et al: Psychometric evaluation of two scales assessing functional status and peripheral neuropathy associated with chemotherapy for ovarian cancer: a Gynecologic Oncology Group study. Oncol Nurs Forum 31: 615-623, 2004.

32. Wenzel L, Huang HQ, Monk BJ, et al: Quality-of-life comparisons in a randomized trial of interval secondary cytoreduction in advanced ovarian carcinoma: a Gynecologic Oncology Group study. J Clin Oncol 23: 5605-5612, 2005.

33. Parmar MK, Ledermann JA, Colombo N, Du Bois A, Delaloye JF, Kristensen GB, Wheeler S, Swart AM, Qian W, Torri V, Floriani I, Jayson G, Lamont A, Tropé C; ICON and AGO Collaborators: Paclitaxel plus platinum-based chemotherapy versus conventional platinum-based chemotherapy in woman with relapsed ovarian cancer: the ICON4/AGO-OVAR-2.2 trial. Lancet 361: 2099-2106, 2003. 